\title{
Structure-activity relationship of a glycolipid, sulfoquinovosyl diacylglycerol, with the DNA binding activity of p53
}

\author{
HIROSHI IIJIMA ${ }^{1}$, NOBUYUKI KASAI ${ }^{2}$, HIROYUKI CHIKU ${ }^{2}$, TOSHIFUMI TAKEUCHI ${ }^{2}$, \\ KOUJI KURAMOCHI ${ }^{2}$, SHINYA HANASHIMA ${ }^{2}$, SUSUMU KOBAYASHI ${ }^{3,4}$, FUMIO SUGAWARA ${ }^{2,4}$, \\ KENGO SAKAGUCHI $^{2,4}$, HIROMI YOSHIDA ${ }^{1,5}$ and YOSHIYUKI MIZUSHINA ${ }^{1,5}$ \\ ${ }^{1}$ Laboratory of Food and Nutritional Sciences, Department of Nutritional Science, Kobe-Gakuin University, \\ Nishi-ku, Kobe, Hyogo 651-2180; ${ }^{2}$ Department of Applied Biological Science, Faculty of Science \\ and Technology; ${ }^{3}$ Faculty of Pharmaceutical Sciences; ${ }^{4}$ Frontier Research Center for Genome and \\ Drug Discovery, Tokyo University of Science, Noda, Chiba 278-8510; ${ }^{5}$ Cooperative Research \\ Center of Life Sciences, Kobe-Gakuin University, Nishi-ku, Kobe, Hyogo 651-2180, Japan
}

Received July 6, 2006; Accepted August 30, 2006

\begin{abstract}
The in vitro relationship between the human $\mathrm{p} 53$ DNA binding domain (p53 DBD) and glycolipids was investigated. We isolated the glycolipid fraction from spinach (Spinacia oleracea L.) and found that the fraction inhibited the double-stranded DNA (dsDNA) binding activity of p53 DBD. Since the fraction contained mainly three glycolipids, monogalactosyl diacylglycerol (MGDG), digalactosyl diacylglycerol (DGDG) and sulfoquinovosyl diacylglycerol (SQDG), and each glycolipid was purified using silica gel column chromatography. Purified SQDG inhibited the activity, however, purified MGDG and DGDG had no influence. In this study, we demonstrated the structure-function relationship between chemically synthetic SQDG and p53 DBD. The major action is probably dependent on the fatty acid effect, although SQDG was a much stronger inhibitor than the fatty acid alone present in SQDG. The inhibitory activity of SQDG was weakened by the R248A mutant of p53 DBD, suggesting that R248 in the dsDNA binding site of p53 must be important for the inhibitory activity of SQDG. SQDG binding to p53 DBD could be reversed with a non-ionic detergent, Nonidet P-40.
\end{abstract}

Correspondence to: Dr Yoshiyuki Mizushina, Laboratory of Food and Nutritional Sciences, Department of Nutritional Science, Kobe-Gakuin University, Nishi-ku, Kobe, Hyogo 651-2180, Japan

E-mail: mizushin@nutr.kobegakuin.ac.jp

Abbreviations: DBD, DNA-binding domain; dsDNA, doublestranded DNA; SQDG, sulfoquinovosyl diacylglycerol; SQMG, sulfoquinovosyl monoacylglycerol; MGDG, monogalactosyl diacylglycerol; DGDG, digalactosyl diacylglycerol; BSA, bovine serum albumin; NP-40, Nonidet P-40; PSII, photosystem II

Key words: glycolipid, sulfoquinovosyl diacylglycerol, p53, DNA-binding activity, inhibitory activity, docking simulation
This is the first study of a glycolipid, SQDG, acting as a dsDNA binding inhibitor of p53, and it could be considered that a SQDG-containing thylakoid membrane in plant chloroplasts might regulate the activity of p53 for cell division, cell cycle checkpoint and tumor suppression.

\section{Introduction}

The tumor suppressor protein p53 (393 amino acids) is a transcription factor that functions to maintain the integrity of the genome (1). On its induction in response to DNA damage, p53 promotes cell cycle arrest in G1 phase (2) and apoptosis if DNA repair is not possible (3). The p53 gene encodes a protein with a central DNA binding domain (DBD) comprised of residues 102-292, flanked by an N-terminal transactivation domain, and a C-terminal tetramerization domain (4). The crystal structure of the core domain (i.e., p53 DBD) bound to DNA has been determined (5). The structure of the DNAbinding domain consists of a large $\beta$-sandwich that acts as a scaffold for three loop-based elements that contact the DNA (5). Importantly, the residues most frequently mutated in cancers are all at or near the protein-DNA interface, and over two-thirds of the missense mutations are within the DNAbinding loops (6). While it is clear that tumorigenic p53-DBD mutations act by diminishing the DNA binding activity of p53 $(7,8)$, the mechanisms are not fully characterized.

We have been screening the natural compounds which inhibited the double-stranded DNA (dsDNA) binding activity of human p53 DBD, because inhibitors can elucidate the structure and function of p53. Recently, we found that the glycolipid fraction from spinach (Spinacia oleracea L.) was a potent inhibitor of p53 DBD activity. This fraction mainly contains the major glycolipids in the classes of monogalactosyl diacylglycerol (MGDG), digalactosyl diacylglycerol (DGDG) and sulfoquinovosyl diacylglycerol (SQDG). Subsequently, we investigated the effects on the inhibitory activity of p53 DBD against purified glycolipids from spinach, and the chemically synthesized the related compounds of the glycolipid. We found that SQDG binds or 
interacts with p53 DBD and strongly suppresses its activity. In this study, we discuss the effects of SQDG on the activity of p53. This study of glycolipids may help to clarify the structure and function of p53-DBD.

\section{Materials and methods}

Materials. Spinach (Spinacia oleracea L.) was purchased in May, 2005 from a supermarket (Co-operative Union Co., Kobe, Hyogo prefecture, Japan). Diaion HP-20 was obtained from Mitsubishi Chemical Inc. (Tokyo, Japan). Supercoiled pBR322 plasmid dsDNA was obtained from Takara Bio Inc. (Kyoto, Japan). The glycolipid, SQDG, and its derivatives were chemically synthesized as described previously (9). All other reagents were of analytical grade and purchased from Nacalai Tesque (Kyoto, Japan).

Extraction and isolation of the glycolipid fraction from spinach. Water-soluble substances were extracted from dried spinach $(50 \mathrm{~g})$ with $1000 \mathrm{ml}$ of warm water $\left(60^{\circ} \mathrm{C}\right)$. The tissue cake was added to $1000 \mathrm{ml}$ of warm ethanol $\left(60^{\circ} \mathrm{C}\right)$, and the substances containing glycolipids were extracted. Ethanol extract $(100 \%)$ was diluted with water to a $70 \%$ ethanol solution. The solution was subjected to Diaion HP-20 column chromatography $(200 \mathrm{ml})$, a hydrophobic type of chromatography, washed with $1000 \mathrm{ml}$ of $70 \%$ ethanol, and then eluted using $95 \%$ ethanol $(500 \mathrm{ml})$. Ethanol solution $(95 \%)$ was the glycolipid fraction.

Purification of glycolipids from spinach. The glycolipid fraction from spinach was applied to silica gel column chromatography and eluted using increasing concentrations of ethyl acetate in chloroform. The fraction containing MGDG was obtained in chloroform/ethyl acetate (5/45, v/v). The column was then successively eluted using increasing concentrations of methanol in ethyl acetate. Fractions A and B were eluted with ethyl acetate/methanol (5/1, v/v) and ethyl acetate/methanol (2/1, v/v), respectively. Fraction A was concentrated and dissolved in a small volume of ethyl acetate. It was then applied to the same silica gel column and eluted using increasing concentrations of acetone in ethyl acetate. Fraction $\mathrm{C}$ was obtained in ethyl acetate/acetone $(1 / 4, \mathrm{v} / \mathrm{v})$, concentrated and dissolved in a small volume of methanol/ water (4/1, v/v), applied to a Sep-Pak C18 column, and eluted using increasing concentrations of methanol in water. The fraction containing DGDG was obtained in methanol. Fraction B was concentrated and dissolved in a small volume of ethyl acetate, and then applied to the same silica gel column. Fraction D was eluted in ethyl acetate/methanol/water (1000/ 200/15, v/v), concentrated and dissolved in a small volume of methanol/water (4/1, v/v), applied to a Sep-Pak C18 column, and eluted using increasing concentrations of methanol in water. The fraction containing SQDG was obtained in methanol/water $(9 / 1, \mathrm{v} / \mathrm{v})$.

${ }^{1} \mathrm{H}-\mathrm{NMR}$ was recorded on a Bruker DRX600. ${ }^{13} \mathrm{C}-\mathrm{NMR}$ was recorded on a DRX400. Chemical shifts were reported in $\delta$, parts per million (ppm), relative to TMS as an internal standard or calibrated using residual undeuterated solvent as an internal reference. IR spectra were recorded on a Jasco FT/IR-410 spectrometer. The structures of MGDG and DGDG

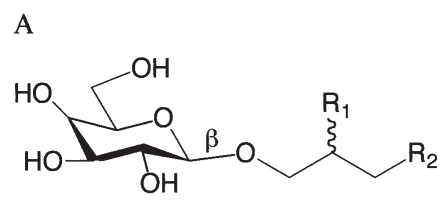

B

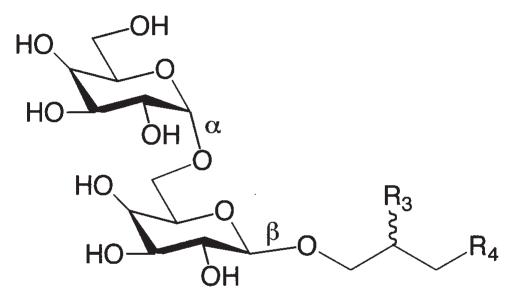

C

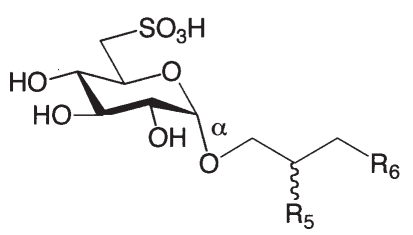

Figure. 1. Chemical structures of the glycolipids from spinach (Spinacia oleracea L.). (A) MGDG (monogalactosyl diacylglycerol). (B) DGDG (digalactosyl diacylglycerol). (C) SQDG (sulfoquinovosyl diacylglycerol). R1 to R6 in these structures are fatty acids.

were identified from the spectral data and by comparison with literature data (10-13). SQDG was identified from the spectral data and by comparison with our data, which we have previously reported (9).

MGDG: ${ }^{1} \mathrm{H}$ NMR $\left(\mathrm{CDCl}_{3}, 600 \mathrm{MHz}\right) \delta$ 5.40-5.30 (13H, m), $4.40(1 \mathrm{H}, \mathrm{dd}, J=11.9 \mathrm{~Hz}, 3.2 \mathrm{~Hz}), 4.28(1 \mathrm{H}, \mathrm{d}, J=7.4 \mathrm{~Hz})$, $4.21(1 \mathrm{H}, \mathrm{dd}, J=11.9 \mathrm{~Hz}, 6.4 \mathrm{~Hz}), 4.01(1 \mathrm{H}, b r m), 3.98(1 \mathrm{H}$, $\mathrm{dd}, J=12.2 \mathrm{~Hz}, 5.8 \mathrm{~Hz}), 3.91(1 \mathrm{H}, \mathrm{dd}, J=11.3 \mathrm{~Hz}, 5.5 \mathrm{~Hz})$, $3.86(1 \mathrm{H}, \mathrm{dd}, J=12.2 \mathrm{~Hz}, 4.0 \mathrm{~Hz}), 3.73(1 \mathrm{H}, \mathrm{m}), 3.66(1 \mathrm{H}, \mathrm{m})$, $3.60(1 \mathrm{H}, \mathrm{dd}, J=9.3 \mathrm{~Hz}, 3.0 \mathrm{~Hz}), 3.55(1 \mathrm{H}, \mathrm{m}), 2.80(8 \mathrm{H}, \mathrm{m})$, 2.32 (4H, m), 2.05 (8H, m), 1.61 (4H, m), 1.40-1.20 (16 H, m), $0.97(6 \mathrm{H}, \mathrm{t}, J=7.6 \mathrm{~Hz}) .{ }^{13} \mathrm{C} \mathrm{NMR}\left(\mathrm{CDCl}_{3}-\mathrm{CD}_{3} \mathrm{OD}, 100 \mathrm{MHz}\right)$ $\delta 173.8,173.4,131.9,130.1,128.2,128.1,127.7,127.0,103.9$, $74.8,73.2,71.3,70.2,69.2,67.9,62.7,61.9,34.1,34.0,29.6$, $29.3,29.1,29.0,29.0,27.1,25.5,25.4,24.7,20.4,14.1$. IR (film) $\mathrm{v}_{\max } 3422,2924,2855,1737,1459,1071 \mathrm{~cm}^{-1}$.

DGDG: ${ }^{1} \mathrm{H}$ NMR $\left(\mathrm{CDCl}_{3}, 600 \mathrm{MHz}\right) \delta$ 5.39-5.24 (13H, m), $4.43(1 \mathrm{H}, \mathrm{dd}, J=12.1 \mathrm{~Hz}, 2.6 \mathrm{~Hz}), 4.23(1 \mathrm{H}, \mathrm{dd}, J=12.0 \mathrm{~Hz}$, $7.3 \mathrm{~Hz}), 4.23(1 \mathrm{H}, \mathrm{d}, J=7.3 \mathrm{~Hz}), 3.93(1 \mathrm{H}, \mathrm{dd}, J=10.1 \mathrm{~Hz}$, $5.3 \mathrm{~Hz}), 3.90-3.87(2 \mathrm{H}, \mathrm{m}), 3.84(1 \mathrm{H}, \mathrm{t}, J=6.0 \mathrm{~Hz}), 3.77$ $(1 \mathrm{H}, \mathrm{dd}, J=10.1 \mathrm{~Hz}, 3.7 \mathrm{~Hz}), 3.74-3.66(8 \mathrm{H}, \mathrm{m}), 3.50(1 \mathrm{H}, \mathrm{dd}$, $J=9.7 \mathrm{~Hz}, 7.3 \mathrm{~Hz}), 3.47(1 \mathrm{H}, \mathrm{dd}, J=3.7 \mathrm{~Hz}, 3.1 \mathrm{~Hz}), 2.80$ $(8 \mathrm{H}, \mathrm{m}), 2.31(4 \mathrm{H}, \mathrm{m}), 2.07(8 \mathrm{H}, \mathrm{m}), 1.60(4 \mathrm{H}, \mathrm{m}), 1.30-1.28$ $(16 \mathrm{H}, \mathrm{m}), 0.97(6 \mathrm{H}, \mathrm{t}, J=7.6 \mathrm{~Hz}) .{ }^{13} \mathrm{C} \mathrm{NMR}\left(\mathrm{CDCl}_{3}-\mathrm{CD}_{3} \mathrm{OD}\right.$, $100 \mathrm{MHz}) \delta 173.8,173.4,131.8,130.1,128.2,128.1,127.7$, 127.0, 103.8, 99.4, 72.9, 72.5, 70.9, 70.3, 70.0, 69.9, 69.9, $69.8,68.7,67.9,66.0,62.7,61.8,34.2,34.0,31.8,29.6,29.5$, $29.3,29.1$,29.1, 29.0, 29.0, 27.1, 25.5, 25.4, 24.8, 24.7, 22.6, 20.4, 14.1. IR (film) $\mathrm{v}_{\max } 3404,2924,2855,1740,1653$, 1461, 1261, 1074, 801, $696 \mathrm{~cm}^{-1}$.

SQDG: ${ }^{1} \mathrm{H}$ NMR $\left(\mathrm{CDCl}_{3}-\mathrm{CD}_{3} \mathrm{OD}, 600 \mathrm{MHz}\right) \delta 5.32(1 \mathrm{H}$, m), $4.41(1 \mathrm{H}, \mathrm{m}), 4.07(2 \mathrm{H}, \mathrm{m}), 3.64(1 \mathrm{H}, \mathrm{m}), 3.43(2 \mathrm{H}, \mathrm{m})$, 


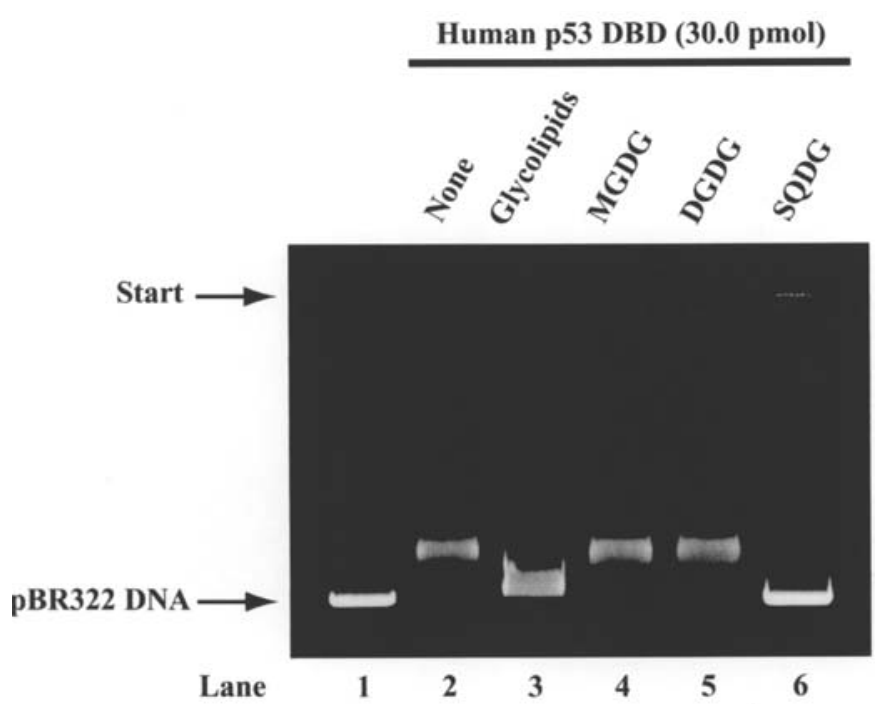

Figure 2. Inhibition of the glycolipids from spinach on the dsDNA binding activity of human p53 DBD. Gel mobility shift assay of binding between pBR322 plasmid dsDNA (0.20 pmol; 870 pmol of nucleotide) and p53 DBD (30.0 pmol, lanes 2-6). Lane 1 contained no proteins. Lanes 3 to 6 contained $400 \mathrm{ng}(20 \mu \mathrm{g} / \mathrm{ml})$ of the glycolipid fraction, purified MGDG, purified DGDG, and purified SQDG, respectively. An ethidium bromide-stained gel is shown.

$3.34(1 \mathrm{H}, \mathrm{m}), 3.15(1 \mathrm{H}, \mathrm{m}), 2.99(1 \mathrm{H}, \mathrm{m}), 2.34(4 \mathrm{H}, \mathrm{m}), 1.60$ $(4 \mathrm{H}, \mathrm{m}), 1.28(56 \mathrm{H}, \mathrm{brm}), 0.89(6 \mathrm{H}, \mathrm{m})$. The chemical structures of the three glycolipids (i.e., MGDG, DGDG and SQDG) are shown in Fig. 1.

Expression and purification of human p53 DBD. The human p53-DNA binding domain (p53 DBD) gene (residues 92-292) was cloned into pET28b between NdeI and XhoI sites. The histidine-tagged protein was overexpressed in Escherichia coli strain BL21(DE3) harboring the expression plasmid constructed in our laboratory. The expression vector of the mutant protein (i.e., R248A) was constructed by QuikChange site-directed mutagenesis kit (Stratagene) and fully sequenced. The mutant protein of p53-DBD was overexpressed in Escherichia coli strain BL21(DE3) harboring the expression plasmid pET28b. After Ni-NTA column (Qiagen) purification, following the procedure recommended by the manufacturer, the mutant proteins were purified on a heparin column (Amersham) by elution with a concentration gradient of $0-1 \mathrm{M}$ potassium chloride.

Gel mobility shift assay. The gel mobility shift assay was carried out as described by Casas-Finet et al (14). The standard binding mixture (a final volume of $20 \mu \mathrm{l}$ ) contained $20 \mathrm{mM}$ Tris-HCl, $\mathrm{pH} 7.5,10 \%$ glycerol, $10 \%$ dimethyl sulfoxide, 1 mM EDTA, 0.20 pmol pBR322 dsDNA (i.e., 870 pmol nucleotide), and $30.0 \mathrm{pmol}(1.50 \mu \mathrm{M})$ human $\mathrm{p} 53 \mathrm{DBD}$. The mixture was incubated for $20 \mathrm{~min}$ on ice. Samples were run on $1.0 \%$ agarose gel in $0.1 \mathrm{M}$ Tris-acetate, $\mathrm{pH} 8.3$, containing $5 \mathrm{mM}$ EDTA at $100 \mathrm{~V}$ for $1 \mathrm{~h}$. The agarose gels were stained with ethidium bromide and dsDNA was visualized on a UV transilluminator. The gel mobility shift length of pBR322 dsDNA was measured with computer software (Zero-D scan, version 1.0, M\&S Instruments Trading).
Structural models of the complex. Molecular docking of human p53 DBD and a glycolipid, SQDG(C18:0) was performed using the Affinity program within Insight II software (Accelrys, San Diego, CA). The coordinates of human p53 DBD (Protein Data Bank (PDB) ID: 1TUP) were obtained from PDB. The initial position of SQDG(C18:0) was determined based on the result of the dsDNA gel mobility shift assay with mutant $\mathrm{p} 53 \mathrm{DBD}$ and the protein surface of the hydrophobicity. The calculation used a CVFF force field in the Discovery program (Accelrys).

\section{Results}

Inhibitory effects of glycolipids from spinach on the dsDNA binding activity of human p53 DBD. We first established the assay method of dsDNA binding activity of human p53 DBD, which is the central region of $\mathrm{p} 53$, using agarose gel electrophoresis. As shown in Fig. 2, 30.0 pmol of p53 DBD bound to pBR322 dsDNA, and p53 DBD and dsDNA were complexed; therefore, the dsDNA stained with ethidium bromide was shifted to the upper side (lane 2). Since the same amount of BSA (bovine serum albumin) instead of p53 DBD did not shift at all (data not shown), dsDNA binding activity could occur selectively to a specific site on p53 DBD. The activity of p53 DBD was dose-dependent, and the distance of the stained and shifted band could be proportional to dsDNA binding activity. We decided the activity by the shifted distances of the band, and 30.0 pmol of p53 DBD (i.e., $1.50 \mu \mathrm{M}$, lane 2) was used in the experiments in this study.

As described in the Introduction, inhibitors of dsDNAbinding activity of human p53 DBD were screened from natural sources. We found that the glycolipid fraction from spinach (Spinacia oleracea L.) potently inhibited activity, and other fractions such as water-soluble and fat-soluble fractions from spinach had no influence. The glycolipid fraction (400 ng; i.e., $20 \mu \mathrm{g} / \mathrm{ml}$ ) moderately inhibited the activity of p53 DBD (lane 3 in Fig. 2). Since this fraction contains three main glycolipids such as MGDG, DGDG and SQDG, and these compounds were completely purified from spinach by silica gel column chromatography. The same amounts (400 ng each) of purified MGDG and DGDG had no influence on the dsDNA binding activity of p53 DBD at all (lanes 4 and 5, respectively), whereas purified SQDG completely inhibited the activity (lane 6). The inhibitory effect of purified SQDG was stronger than that of the same amount of the glycolipid fraction from spinach. These results suggested that SQDG in the glycolipid fraction must be an inhibitory compound of activity. Therefore, we concentrated SQDG and its derivatives, which were chemically synthesized, in the latter part of this study.

Inhibitory effects of synthetic SQDG and its derivatives on the activity of human $553 \mathrm{DBD}$. SQDG can be separated into four chemical parts: monosaccharide (i.e., D-glucose, Fig. 3D) (Group I), a sulfate moiety (Group II), glycerol (Fig. 3F) (Group III) and fatty acid(s) (i.e., stearic acid (C18:0), Fig. 3H) (Group IV). Since the method of chemical synthesis of SQDG succeeded previously (9), SQDG (Groups I-IV) was synthesized by assembling the derivatives of each of the four parts into different kinds of sulfoquinovosyl acylglycerols. The 
A

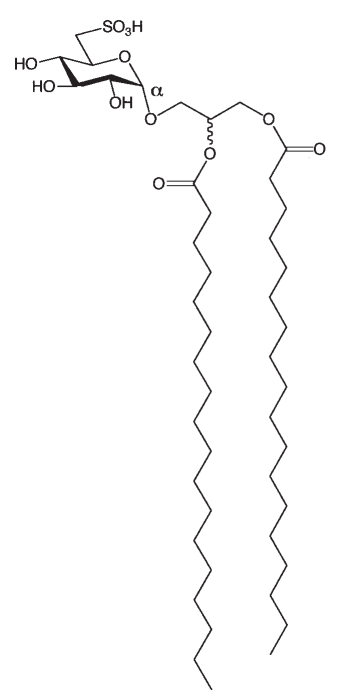

C

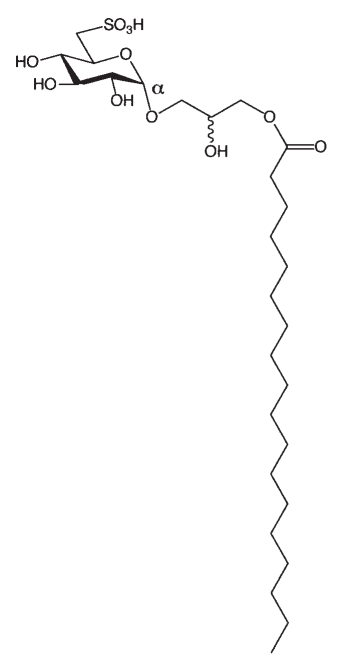

G

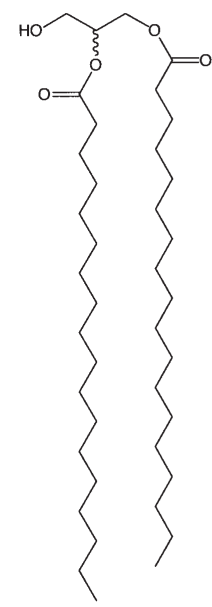

B

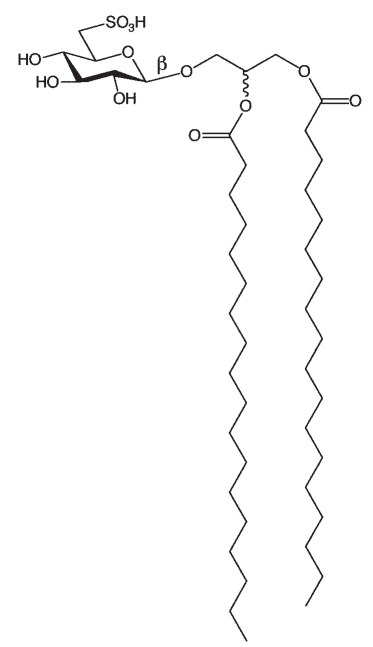

D

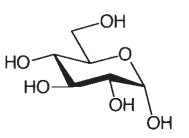

E

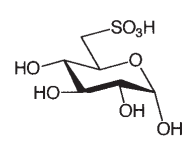

$\mathrm{F}$

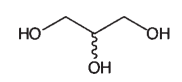

$\mathrm{H}$

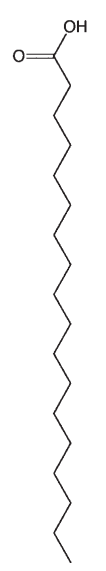

Figure 3. Chemically synthesized structures of derivatives and stereoisomers of SQDG. SQDG separated into four groups. Group I: Monosaccharide, Group II: 6-Sulfate moiety, Group III: Glycerol, Group IV: Fatty acid(s). (A) 1,2-di-O-acyl-3-O-(6-deoxy-6-sulfo- $\alpha$-D-glucopyranosyl)-sn-glycerol ( $\alpha$-SQDG(C18:0), Groups I-IV). (B) 1,2-di-O-acyl-3-O-(6-deoxy-6-sulfo- 3 D-glucopyranosyl)-sn-glycerol (ß-SQDG(C18:0), Groups I-IV). (C) 1-mono$O$-acyl-3-O-(6-deoxy-6-sulfo- $\alpha$-D-glucopyranosyl)-sn-glycerol ( $\alpha$-SQMG (C18:0), Groups I-IV). (D) D-glucose (Group I). (E) D-glucose-6-sulfate (Groups I and II). (F) Glycerol (Group III). (G) 1,2-Diacylglycerol (Groups III and IV). (H) Stearic acid (C18:0, Group IV).

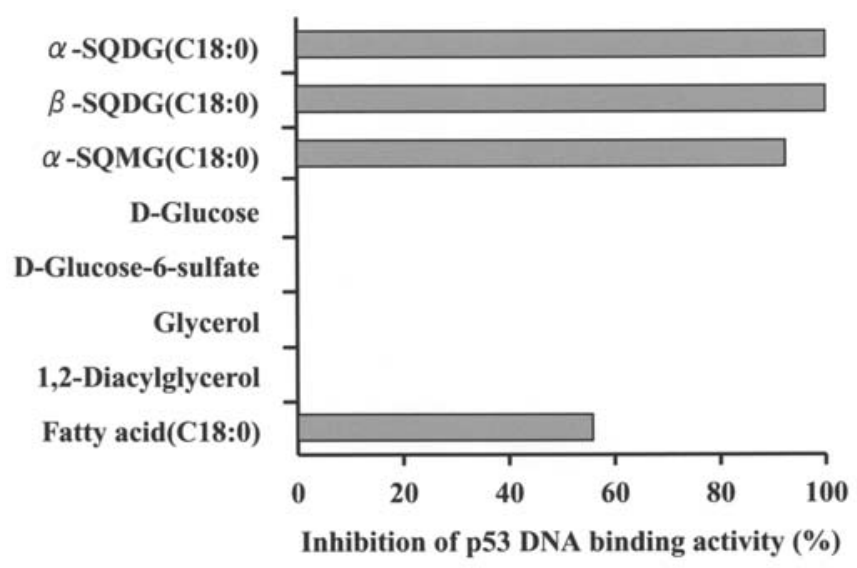

Figure 4. Inhibition of synthesized SQDG and its derivatives (2.4 nmol each) on the dsDNA binding activity of human p53 DBD. The dsDNA binding activity of p53 DBD (30.0 pmol) in the absence of compounds was taken as $0 \%$.

basic structures of synthesized SQDG $[\alpha-\operatorname{SQDG}(\mathrm{C} 18: 0)]$ are shown in Fig. 3A, consisting of two fatty acids [i.e., C18saturated fatty acid, stearic acid (C18:0)]. Sulfoquinovosyl monoacylglycerol (SQMG), which consists of one fatty acid (Groups I-IV), was also synthesized [Fig. 3C, $(\alpha-S Q M G$ (C18:0)]. SQMG/SQDG could have stereoisomers of two configurations, $\alpha$ - or $\beta$-type, between sulfoquinovose (Groups I and II) and glycerol (Group III). SQDG/SQMG of $\alpha$-configuration (Fig. 3A and $\mathrm{C}$ ) is the natural type, and SQDG of B-configuration [Fig. 3B, (ß-SQDG(C18:0)] is the unnatural type (9). Parts of SQDG compounds of such as D-glucose6-sulfate (Fig. 3E) (Groups I and II) and 1,2-diacylglycerol (Fig. 3G) (Groups III and IV) were also prepared.

As shown in Fig. 4, $2.4 \mathrm{nmol}(120 \mu \mathrm{M})$ of synthesized $\alpha-S Q D G(C 18: 0)$ completely inhibited the dsDNA binding activity of human p53 DBD. B-SQDG(C18:0) also inhibited the activity to a similar extent as $\alpha-\mathrm{SQDG}(\mathrm{C} 18: 0)$, suggesting that the configuration is not essential for the inhibitory effect. D-glucose (monosaccharide, Group I), D-glucose-6-sulfate (monosaccharide with a sulfate moiety, Groups I and II), glycerol (Group III), and 1,2-diacylglycerol (Groups III and IV) showed no inhibitory effect.

Stearic acid (C18:0) (fatty acid, Group IV) moderately inhibited the activity of p53 DBD with $57 \%$ inhibition by $2.4 \mathrm{nmol}$ of stearic acid (Fig. 4). The inhibitory effect of $\alpha-S Q M G(C 18: 0)$ also inhibited activity; however, the inhibitory effect of $\alpha-S Q M G(C 18: 0)$ was slightly weaker than that of $\alpha$-SQDG(C18:0). These results suggested that fatty acid moiety and its number of SQDG/SQMG might be important for inhibition.

The sulfate moiety (Group II) in the monosaccharide was essential for inhibition, since neutral glycolipids such as MGDG and DGDG, consisting of Groups I, III and IV, showed no inhibitory effect (Fig. 2). On the other hand, $2.4 \mathrm{nmol}$ of cholesterol sulfate inhibited the dsDNA activity of p53 DBD, and cholesterol had no influence on the activity at the same concentration (data not shown), suggesting that the sulfate moiety of the anionic lipids was important. The inhibitory effect was strongly influenced by partial change of the SQDG structure, suggesting that stronger inhibitory protocols for the 
A

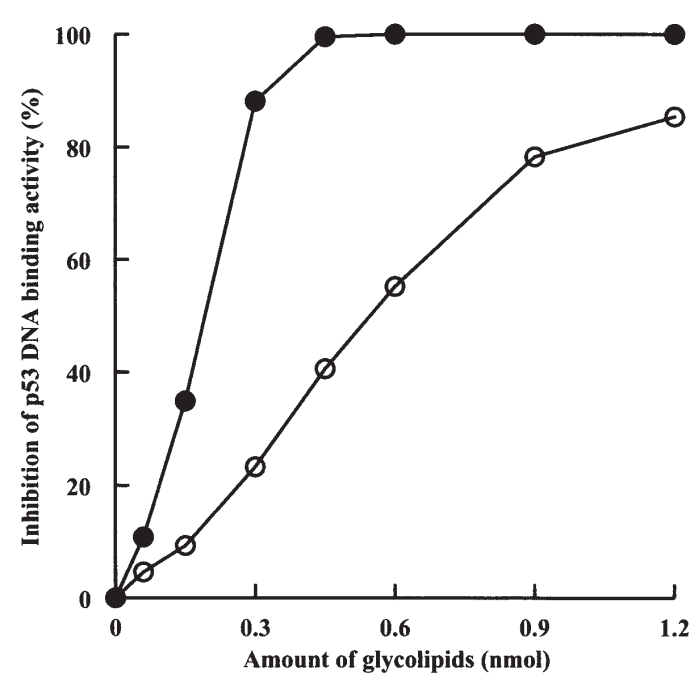

B

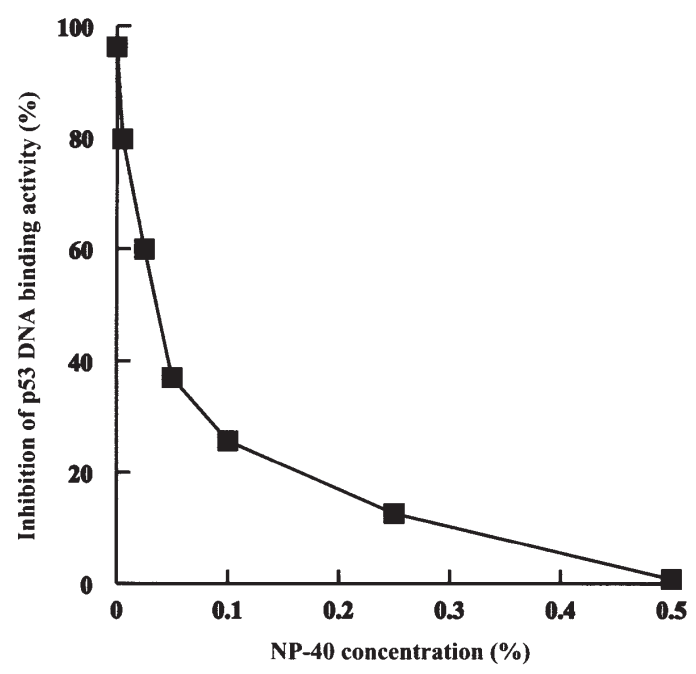

Figure 5. Inhibitory effect of synthesized SQDG/SQMG on the dsDNA binding activity of human p53 DBD. (A) Inhibitory dose-response curves of $\alpha$-SQDG(C18:0) (closed circle) and $\alpha$-SQMG(C18:0) (open circle). (B) Effect of detergents on the inhibition of human p53 DBD activities by $\alpha-\operatorname{SQDG}(\mathrm{C} 18: 0), \alpha-\operatorname{SQDG}(\mathrm{C} 18: 0)$ was present at a concentration of $0.60 \mathrm{nmol}$, and Nonidet P-40 (NP-40) was added to the reaction mixture at $0-0.5 \%$. The dsDNA binding activity of $\mathrm{p} 53 \mathrm{DBD}(30.0 \mathrm{pmol})$ in the absence of SQDG/SQMG was taken as $0 \%$.

synthesis of new lipids containing sulfate (i.e., sulfolipids) are possible.

Effects of reaction conditions on $\alpha-S Q D G / S Q M G(C 18: 0)$ inhibition. Fig. 5A shows the inhibition dose-response curves of the synthesized $\alpha$-SQDG/SQMG(C18:0) against human p53 DBD (30.0 pmol). Both $\alpha$-SQDG(C18:0) and $\alpha-S Q M G(C 18: 0)$ were effective at inhibiting the dsDNA binding activity of p53 DBD, with 50\% inhibition observed at $0.18 \mathrm{nmol}(9.0 \mu \mathrm{M})$ and $0.55 \mathrm{nmol}(27.5 \mu \mathrm{M})$, respectively. The inhibitory effect of $\alpha$-SQDG(C18:0) was $\sim 3$-fold stronger than that of $\alpha$-SQMG (C18:0). The synthesized B-SQDG(C18:0), which is an unnatural stereoisomer of $\alpha-\operatorname{SQDG}(\mathrm{C} 18: 0)$, inhibited to the same extent as $\alpha$-SQDG(C18:0), with 50\% inhibition of

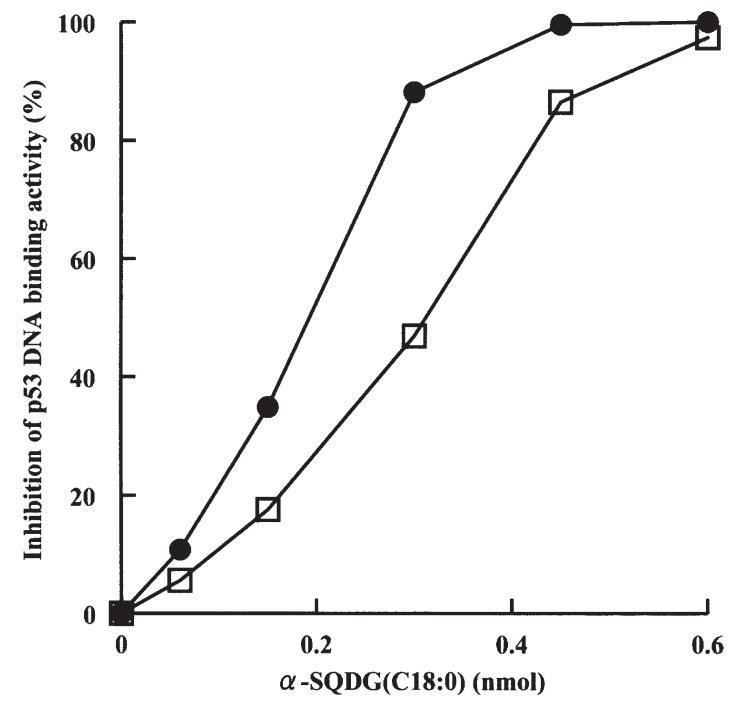

Figure 6. Effect of $\alpha$-SQDG(C18:0) on the inhibition of R248A mutant of human p53 DBD activity. Inhibitory dose-response curves of $\alpha$-SQDG (C18:0) for the dsDNA binding activities of p53 DBD wild-type (30.0 pmol, closed circle) or R248A mutant (30.0 pmol, open square). The dsDNAbinding activity of p53 DBD (30.0 pmol) in the absence of $\alpha$-SQDG(C18:0) was taken as $0 \%$.

$0.19 \mathrm{nmol}(9.5 \mu \mathrm{M})$ (data not shown). Therefore, we concentrated $\alpha-\operatorname{SQDG}(\mathrm{C} 18: 0)$, which was chemically synthesized, in the latter part of this study.

To determine the effects of a non-ionic detergent on the binding of $\alpha$-SQDG(C18:0) and human p53 DBD, Nonidet P-40 (NP-40) was added to the reaction mixture at various concentrations. The p53 DBD inhibitory effect of $\alpha$-SQDG (C18:0) at $0.60 \mathrm{nmol}$ was significantly reversed by the addition of NP-40 to the reaction mixture (Fig. 5B). The inhibitory reversion by NP-40 was concentration dependent, and the reversion of $>50 \%$ was found by the addition of $0.5 \%$ NP- 40 . This result suggested that the fatty acid moiety of $\alpha$-SQDG (C18:0) might interact with the hydrophobic region of p53 DBD protein.

We also tested whether an excess amount of a DNA analog, poly $(\mathrm{rC})(2.00 \mathrm{pmol})$, or a protein, BSA $(12.5 \mu \mathrm{M}, 250 \mathrm{pmol})$, could prevent the inhibitory effects of $\alpha-\operatorname{SQDG}(\mathrm{C} 18: 0)$. If $\alpha-S Q D G(C 18: 0)$ binds to 553 DBD by non-specific adhesion, the addition of a nucleic acid and/or protein would be expected to reduce inhibitory activity. The fact that neither poly(rC) nor BSA influenced the inhibitory effects of $\alpha-\operatorname{SQDG}(\mathrm{C} 18: 0)$ suggests that the compound occurs selectively or binds to a specific site on 553 DBD and not to the nucleic acid.

Binding analysis of $\alpha-S Q D G(C 18: 0)$ and human $p 53$ DBD. To determine whether SQDG resulted in binding to DNA or human p53 DBD, the interaction of SQDG with dsDNA was investigated based on the thermal transition of dsDNA with or without $\alpha$-SQDG(C18:0). The melting temperature (Tm) of dsDNA with an excess amount of $\alpha$-SQDG(C18:0) (100 $\mu \mathrm{M})$ was measured using a spectrophotometer equipped with a thermoelectric cell holder. In the concentration range used, no thermal transition of Tm was observed, whereas when ethidium bromide, a typical intercalating compound, was used as a 
A

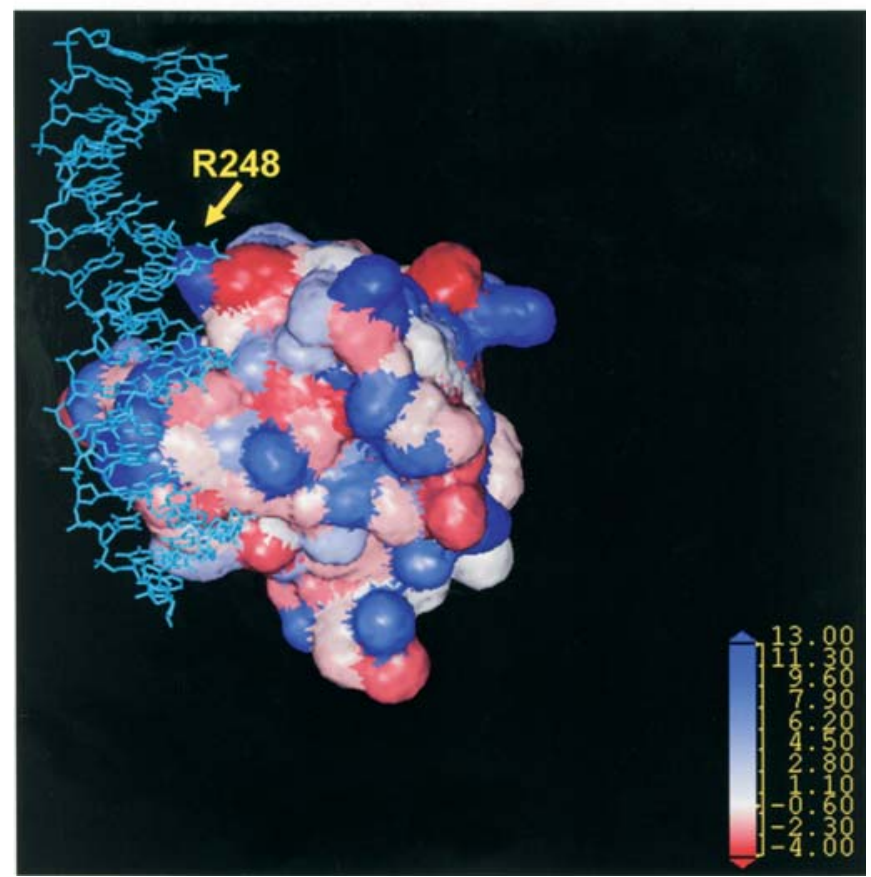

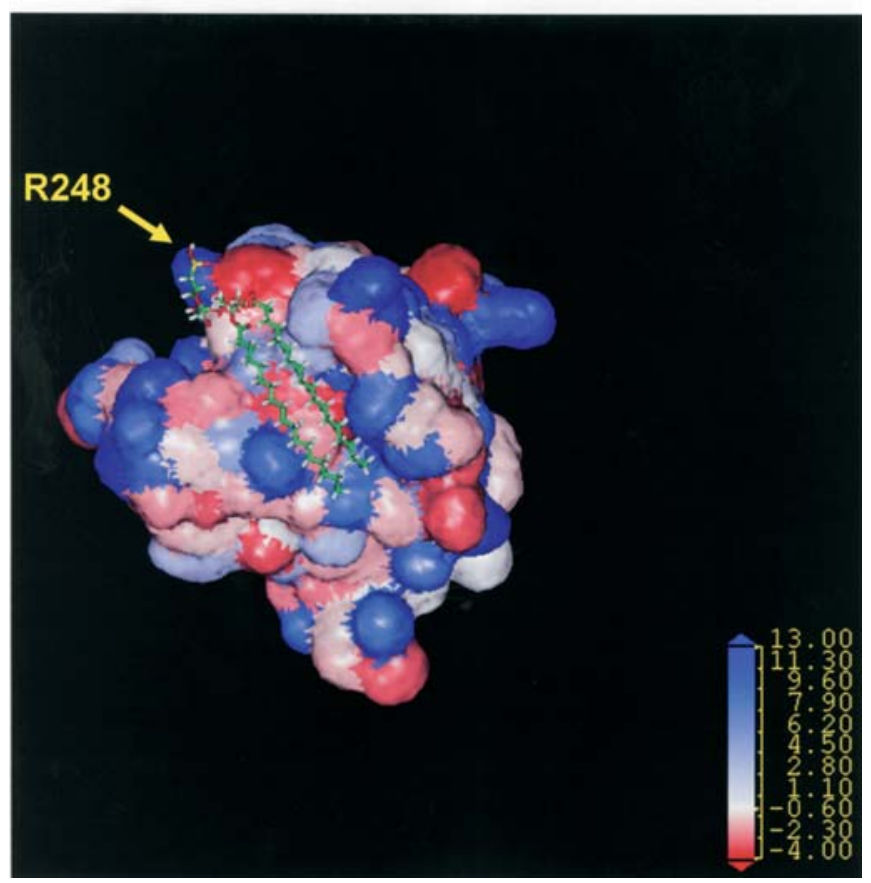

Figure 7. Simulation of the $\alpha$-SQDG(C18:0) interaction interface on human p53 DBD. (A) The complex of dsDNA and p53 DBD. (B) The complex of $\alpha$-SQDG(C18:0) and p53 DBD. Connolly surface and electrostatic potential of p53 DBD are shown, blue is a positive charge, and red is negative. The carbons, oxygens, hydrogens and sulfurs of the structure of $\alpha$-SQDG(C18:0) is indicated in green, red, white and orange, respectively. The molecular docking of the DNA binding domain of human p53 DBD (PDB: 1TUP) was performed using the Affinity program within Insight II software. The calculation used a CVFF force field in the Discovery program.

positive control, it produced clear thermal transition (data not shown). The results indicated that this compound did not intercalate to dsDNA, and fatty acid might directly bind to p53 DBD and inhibit its activity.

To confirm direct binding between SQDG and human p53 $\mathrm{DBD}$, the kinetic parameters for $\alpha-S Q D G(C 18: 0)$ and binding were determined using p53 DBD immobilized to the sensor chip in a BIAcore. p53 DBD (565 pmol) was conjugated to the CM5 sensor chip, and $\alpha-S Q D G(C 18: 0)$ was added to the conjugated proteins. The response differences for the binding of $\alpha$-SQDG $(\mathrm{C} 18: 0)$ to the protein were $\sim 200 \mathrm{RU}$, and this compound dissociated very slowly from p53 DBD. The dissociation constants $\left(\mathrm{K}_{\mathrm{D}}\right)$ of the binding of $\alpha$-SQDG(C18:0) to the protein were determined to be $5.41 \mathrm{nM}$ from these data. Kinetic studies supported the suggestion that $\alpha-\operatorname{SQDG}(\mathrm{C} 18: 0)$ interacted with p53 DBD directly.

Inhibitory effect of $\alpha-S Q D G(C 18: 0)$ on the dsDNA binding activity of the mutant of human $553 \mathrm{DBD}$. Sequencing of the p53 gene in mammals, amphibians, birds, and fish has revealed five highly conserved domains, four of which fall within exons 5 through 8 (15): domain II (residues 117-142), domain III (residues 171-181), domain IV (residues 234-258), and domain V (residues 270-286). p53 is mutated in over half of all tumors. The overwhelming majority of these changes are found in the central DNA binding domain (i.e., p53 DBD) comprised of residues 94-312, which consists of domains II to V (5). Six tumorigenic mutations (R175, G245, R248, R249, $\mathrm{R} 273$ and R282) are most prevalent in the general population
$(5,16)$, and these positions account for $22 \%$ of the 15000 p53 mutations identified from human tumors and cell lines according to the database compiled by the Institut Curie (http://p53.free.fr/). In particular, R248 in domain IV is the most common mutation (17); therefore, an R248A mutant of human p53 DBD was prepared, and the inhibitory effect of fatty acids was investigated as compared with the wildtype.

Fig. 6 shows the inhibition dose-response curves of $\alpha-S Q D G(C 18: 0)$ against the wild-type and R248A mutant of human p53 DBD (30.0 pmol). The compound was effective at inhibiting the dsDNA binding activity of both wild-type and mutant p53 DBD, although inhibition of the activity of mutant p53 DBD by $\alpha$-SQDG(C18:0) was 1.77 -fold weaker than that of the wild-type. These results suggested that $\alpha-S Q D G(C 18: 0)$ must bind to or interact with R248 on the dsDNA binding surface of p53 DBD.

Simulation of the docking of human p53 DBD with a-SQDG (C18:0). As described above, the three-dimensional structure of human p53 DBD with or without chemically synthesized SQDG, $\alpha-$ SQDG(C18:0), was studied (Fig. 7). The crystal structure of human p53 DBD in complex with consensus dsDNA is shown in Fig. 7A. A large immunoglobulin-like $ß$-sandwich, forming a compact barrel-like structure, provides the basic scaffold for the conserved DNA binding surface (the blue area of p53 DBD in Fig. 7). This extended surface, rich in basic amino acids, consists of a loop-sheet-helix motif and two large loops (L2 and L3). 
The $\alpha$-SQDG(C18:0) binding site of p53 DBD was refined using Insight II/Discovery (Accelrys) (Fig. 7B). A cavity (29 $\AA$ long, $8 \AA$ wide and $3 \AA$ high, respectively) where SQDG with two stearic acids (C18:0) can bind was searched for on the surface of the protein, and found in the hydrophobic area. $\alpha$-SQDG(C18:0) was suggested to interact with the hydrophobic region of p53 DBD, because the complex of $\alpha$-SQDG (C18:0) and p53 DBD was reversed by a neutral detergent, NP-40 (Fig. 5B). In these simulation results, the sulfate moiety of $\alpha$-SQDG(C18:0) on p53 DBD was in the same position as the minor groove of dsDNA on $\mathrm{p} 53 \mathrm{DBD}$, because the free sulfate group of $\alpha-S Q D G(C 18: 0)$ was required for the inhibition of dsDNA binding activity (Fig. 4). Furthermore, the inhibitory effect of the compound on the R248A mutant of p53 DBD was weaker than on the wild-type (Fig. 6), the acidic group of the sulfate moiety of SQDG could interact with the basic residue of R248, and the alkyl chain region of the fatty acid moiety of SQDG interacted with the hydrophobic surface of p53. It was suggested that SQDG bound to the residue of R248 of p53 DBD and inhibited dsDNA binding activity by competing with dsDNA. Since the inhibitory effects of $\beta-S Q D G(C 18: 0)$, which is a stereoisomer of $\alpha-S Q D G$ (C18:0) and synthetic compound, on the dsDNA binding activity of both wild-type and mutant p53 DBD was as strong as those of $\alpha$-SQDG(C18:0), it could be considered that B-SQDG(C18:0) binding pockets in 553 DBD could be in the same position as the $\alpha$-SQDG(C18:0) binding region. The size of the pockets may be a key to explain these inhibition characteristics by the length of SQDG fatty acids. This is the first study to find that SQDG could bind to human p53 directly, and inhibit or regulate the dsDNA binding activity of $\mathrm{p} 53$.

\section{Discussion}

The lipid composition of thylakoid membranes is highly conserved among higher plants, algae, and cyanobacteria, comprised mainly of the following three glycolipids, MGDG, DGDG, and SQDG (18). MGDG and DGDG are noncharged lipids, whereas SQDG possesses a negatively charged head group. Thylakoid membranes in plant chloroplasts and cyanobacterial cells are unique in possessing photosynthetic electron transport and photophosphorylation systems for the conversion of light to chemical energy. A mutant of Chlamydomonas reinhardtii, defective in SQDG ( $h f-2)$, showed photosystem II (PSII) activity that was $40 \%$ lower than that of the wild-type, an increase in sensitivity of the PSII activity to 3-(3,4-dichlorophenyl)-1,1-dimethylurea (DCMU), and a lower growth rate (19-22). In accordance with these observations, the incubation of isolated thylakoid membranes of $h f-2$ with SQDG in vitro reversed the lowered PSII activity. Therefore, these results concluded that SQDG has the specific function of maintaining PSII properties.

The p53 tumor suppressor protein responds to a variety of cellular stresses, including DNA damage, UV irradiation, hypoxia and aberrantly activated oncogenes, and may induce cell-cycle arrest or apoptosis (23-25). However, the molecular regulation mechanisms of the various biological activities of p53 have not been characterized. In this study, we found that SQDG, which is an acidic glycolipid, strongly inhibited the
dsDNA binding activity of human p53 DBD. Plant glycolipids, especially SQDG, might regulate the biological activities of p53 to achieve complexes of SQDG and p53 in vivo. The in vivo regulation mechanism of $\mathrm{p} 53$ by SQDG in plants will be a future study.

\section{Acknowledgements}

This work was supported in part by a Grant-in-aid for KobeGakuin University Joint Research (A), and 'Academic Frontier' Project for Private Universities: matching fund subsidy from MEXT (Ministry of Education, Culture, Sports, Science and Technology), 2006-2010, (H.Y. and Y.M.). Y.M. acknowledges Grants-in-aid from the Mochida Memorial Foundation for Medical and Pharmaceutical Research (Japan), and the Nakashima Foundation (Japan).

\section{References}

1. Lane DP: Cancer. p53, guardian of the genome. Nature 358: $15-16,1992$.

2. Kastan MB, Onyekwere O, Sidransky D, Vogelstein B and Craig RW: Participation of 553 protein in the cellular response to DNA damage. Cancer Res 51: 6304-6311, 1991.

3. Polyak K, Xia Y, Zweier JL, Kinzler KW and Vogelstein B: A model for p53-induced apoptosis. Nature 389: 300-305, 1997.

4. Levine AJ: p53, the cellular gatekeeper for growth and division. Cell 88: 323-331, 1997.

5. Cho Y, Gorina S, Jeffrey PD and Pavletich NP: Crystal structure of a p53 tumor suppressor-DNA complex: understanding tumorigenic mutations. Science 265: 346-355, 1994.

6. Vogelstein B and Kinzler KW: Tumour-suppressor genes. X-rays strike p53 again. Nature 370: 174-175, 1994.

7. Kern SE, Pietenpol JA, Thiagalingam S, Seymour A, Kinzler KW and Vogelstein B: Oncogenic forms of p53 inhibit p53-regulated gene expression. Science 256: 827-830, 1992.

8. Unger T, Nau MM, Segal S and Minna JD: p53: a transdominant regulator of transcription whose function is ablated by mutations occurring in human cancer. EMBO J 11: 1383-1390, 1992.

9. Hanashima S, Mizushina Y, Yamazaki T, Ohta K, Takahashi S, Sahara H, Sakaguchi K and Sugawar F: Synthesis of sulfoquinovosylacylglycerols, inhibitors of eukaryotic DNA polymerase $\alpha$ and $\beta$. Bioorg Med Chem 9: 367-376, 2001.

10. Murakami A, Nakamura Y, Koshimizu K and Ohigashi H: Glyceroglycolipids from Citrus hystrix, a traditional herb in Thailand, potently inhibit the tumor-promoting activity of 12-Otetradecanoylphorbol 13-acetate in mouse skin. J Agric Food Chem 43: 2779-2783, 1995.

11. Yamauchi R, Aizawa K, Inakuma T and Kato K: Analysis of molecular species of glycolipids in fruit pastes of red bell pepper (Capsicum annuum L.) by high-performance liquid chromatography-mass spectrometry. J Agric Food Chem 49: 622-627, 1995.

12. Sakano Y, Mutsuga M, Tanaka R, Suganuma H, Inakuma T, Toyoda M, Goda Y, Shibuya M and Ebizuka Y: Inhibition of human lanosterol synthase by the constituents of Colocasia esculenta (taro). Biol Pharm Bull 28: 299-304, 2005.

13. Wegener $\mathrm{C}$, Hamburger $\mathrm{M}$, Kunert $\mathrm{O}$ and Haslinger E: Tensioactive compounds from the aquatic plant Ranunculus fluitans L. (Ranunculaceae) Helv. Chimica Acta 83: 1454-1464, 2000.

14. Casas-Finet JR, Kumar A, Morris G, Wilson SH and Karpel RL: Spectroscopic studies of the structural domains of mammalian DNA B-polymerase. J Biol Chem 266: 19618-19625, 1991.

15. Soussi T, Caron de Fromentel C and May P: Structural aspects of the p53 protein in relation to gene evolution. Oncogene 5: 945-952, 1990.

16. Hainaut $P$ and Hollstein M: $p 53$ and human cancer: the first ten thousand mutations. Adv Cancer Res 77: 81-137, 2000.

17. Hollstein M, Sidransky D, Vogelstein B and Harris CC: p53 mutations in human cancers. Science 253: 49-53, 1991.

18. Benning C: Biosynthesis and function of the sulfolipid sulfoquinovosyl diacylglycerol. Annu Rev Plant Physiol Plant Mol Biol 49: 53-75, 1998. 
19. Minoda A, Sato N, Nozaki H, Okada K, Takahashi H, Sonoike K and Tsuzuki M: Role of sulfoquinovosyl diacylglycerol for the maintenance of photosystem II in Chlamydomonas reinhardtii. Eur J Biochem 269: 2353-2358, 2002.

20. Sato N, Aoki M, Maru Y, Sonoike K, Minoda A and Tsuzuki M: Involvement of sulfoquinovosyl diacylglycerol in the structural integrity and heat-tolerance of photosystem II. Planta 217: 245-251, 2003

21. Sato N, Tsuzuki M, Matsuda Y, Ehara T, Osafune T and Kawaguchi A: Isolation and characterization of mutants affected in lipid metabolism of Chlamydomonas reinhardtii. Eur J Biochem 230: 987-993, 1995.
22. Sato N, Sonoike K, Tsuzuki M and Kawaguchi A: Impaired photosystem II in a mutant of Chlamydomonas reinhardtii defective in sulfoquinovosyl diacylglycerol. Eur J Biochem 234: 16-23, 1995.

23. Vogelstein B, Lane D and Levine AJ: Surfing the p53 network. Nature 408: 307-310, 2000

24. Ryan KM, Phillips AC and Vousden KH: Regulation and function of the p53 tumor suppressor protein. Curr Opin Cell Biol 13: 332-337, 2001

25. Vousden $\mathrm{KH}$ and Lu X: Live or let die: the cell's response to p53. Nat Rev Cancer 2: 594-604, 2002. 